ARTICLE

\title{
3D microstructure design of lithium-ion battery electrodes assisted by X-ray nano-computed tomography and modelling
}

Xuekun Lu, ${ }^{1,3,7}$, Antonio Bertei ${ }^{4,7}$, Donal P. Finegan (10 5, Chun Tan (10) 1,3, Sohrab R. Daemi', Julia S. Weaving ${ }^{1}$, Kieran B. O'Regan (1) 3,6, Thomas M. M. Heenan (1) 1,3, Gareth Hinds², Emma Kendrick (D) 3,6, Dan J. L. Brett ${ }^{1,3}$ \& Paul R. Shearing $1,3 \times$

Driving range and fast charge capability of electric vehicles are heavily dependent on the 3D microstructure of lithium-ion batteries (LiBs) and substantial fundamental research is required to optimise electrode design for specific operating conditions. Here we have developed a full microstructure-resolved 3D model using a novel $X$-ray nano-computed tomography (CT) dual-scan superimposition technique that captures features of the carbonbinder domain. This elucidates how LiB performance is markedly affected by microstructural heterogeneities, particularly under high rate conditions. The elongated shape and wide size distribution of the active particles not only affect the lithium-ion transport but also lead to a heterogeneous current distribution and non-uniform lithiation between particles and along the through-thickness direction. Building on these insights, we propose and compare potential graded-microstructure designs for next-generation battery electrodes. To guide manufacturing of electrode architectures, in-situ X-ray CT is shown to reliably reveal the porosity and tortuosity changes with incremental calendering steps.

\footnotetext{
${ }^{1}$ Electrochemical Innovation Lab, Department of Chemical Engineering, University College London, London WC1E 7JE, UK. ${ }^{2}$ National Physical Laboratory, Teddington, Middlesex TW11 OLW, UK. ${ }^{3}$ The Faraday Institution, Quad One, Harwell Science and Innovation Campus, Didcot OX11 ORA, UK. ${ }^{4}$ Department of Civil and Industrial Engineering, University of Pisa, 56122 Pisa, Italy. ${ }^{5}$ National Renewable Energy Laboratory, 15013 Denver West Parkway, Golden, CO 80401, USA. ${ }^{6}$ School of Metallurgy and Materials, University of Birmingham, Birmingham B15 2TT, UK. ${ }^{7}$ These authors contributed equally: Xuekun Lu, Antonio Bertei. ${ }_{\text {email: p.shearing@ucl.ac.uk }}$
} 
ithium-ion batteries (LiBs) are the leading energy storage technology for portable electronics and electric vehicles $(E V s)^{1}$, which could alleviate reliance on fossil fuels. However, major challenges in safety, energy and power density, cost and durability of LIBs remain ${ }^{2-4}$ : this study aims to provide prospective solutions to these challenges.

The first challenge this work will address is elucidating the optimum electrode design for specific operating conditions. $\mathrm{LiB}$ electrodes exhibit complex interplay between multiple electrochemically coupled transport processes, which rely upon the microstructure of the constituent phases and their spatial arrangement ${ }^{5-7}$. Comprehensive understanding of the physical and electrochemical processes at the micro-scale is critical to rationalise the microstructural engineering strategy (e.g. porosity, thickness and mass loading) for different applications ${ }^{8-10}$. For instance, the porosity and pore structure are predominantly determined by calendering process, which effectively reduces electrical resistance and improves energy density of the electrode at the cost of rate capability. However, few studies unravel the dependence of tortuosity and porosity on calendering in real time and whether or not it is appropriate to apply the same calendering strategy to electrodes of different thickness.

The second challenge is to understand the effect of heterogeneous electrochemical, mass transport and mechanical properties, on the long-term durability, safety and electrochemical performance of LIBs ${ }^{11-13}$. Heterogeneous pore phase and particle packing can result in high current densities locally, leading to a non-uniform state-of-lithiation $(\mathrm{SoL})^{11,14}$ and hot-spot formation ${ }^{13,15}$. Consequently, uneven utilisation of the active material as well as non-uniform mechanical (cracking and delamination) ${ }^{16-18}$ and electrochemical (ionic mixing and phase transition) aging ${ }^{19,20}$ significantly reduce the cell's cycle life, energy density and safety ${ }^{21,22}$, particularly under high-rate conditions ${ }^{23,24}$. Improved understanding of the interplay between microstructural heterogeneities and battery performance not only helps to alleviate degradation, but also provides new insights into advanced structure design.

Mapping the electrode microstructure in $3 \mathrm{D}$ is necessary to evaluate microstructural heterogeneities and their effect on battery performance, for which X-ray computed tomography (X-ray CT) has emerged as a valuable technique ${ }^{17,25}$. However, X-ray CT of $\mathrm{LiB}$ electrodes is restricted due to the challenge of identifying the carbon-binder domain (CBD). Previously, the pore and CBD were treated as a single phase (pore-CBD phase) that was assumed to be filled with electrolyte ${ }^{23,26}$, which may lead to unrepresentative microstructures and mass transport performance. To accurately correlate the microstructure with performance, a more practical solution is required.

This study aims to drive the advancement of battery performance in the perspectives of both electrode design and manufacturing. Assisted by a novel dual-scan superimposition (DSS) technique that combines the separately imaged low-attenuating $\mathrm{CBD}$ and high-attenuating $\mathrm{LiNi}_{1 / 3} \mathrm{Mn}_{1 / 3} \mathrm{Co}_{1 / 3} \mathrm{O}_{2}$ positive electrode (NMC111), the full $3 \mathrm{D}$ microstructure is reconstructed, metrologically characterised and modelled, by a $3 \mathrm{D}$, microstructure-resolved physics-based battery model of high fidelity, to elucidate the interplay between the microstructure and the electrochemical performance, in order to highlight the effect of heterogeneity on electrochemical-state variables such as SoL, charge transfer, electrolyte concentration and electrochemical potential, both globally and locally. Building on these insights, we propose and compare potential graded-microstructure designs for next-generation Li-ion cells. Finally, in situ X-ray computed tomography is conducted to investigate the microstructural evolution, porosity and tortuosity variation at incremental calendering steps to guide the manufacturing process.

\section{Results}

3D characterisation of microstructural heterogeneities. Lithium-ion battery cells are composed of structural constituents spanning over multiple length scales. Figure 1a shows a typical cylindrical LiB scanned by X-ray micro-computed tomography (micro-CT) at a voxel size of $12.9 \mu \mathrm{m}$, with a corner-cut-out showing the auxiliary components in the cell cap and the internal structure of the cell. The double-sided coated current collector, cathode, separator and anode are wound round the central supportive core, forming periodic layers in the radial direction (Fig. 1b). X-ray nano-CT was then conducted to capture the 3D microstructural details of the layered cell separately prepared by doctor blade coating method (Fig. 1c). Distinct morphologies in terms of particle shape, orientation and pore size are observed in the graphite anode, polyolefin separator and NMC cathode (from left to right). Further magnification of the cathode microstructure was achieved by SEM imaging (Fig. 1d), in which the microstructural details of the CBD, micro and macro-pores, NMC secondary and primary particles of different orientations are resolved. It is noted that X-ray CT failed to reconstruct and distinguish the microstructure details of the CBD and the micropores, which appear as a blurry mixed phase (Fig. 1e), due to the nano-features and the similarly low X-ray attenuation coefficients of carbon and the pores. In addition, the highly X-ray absorbing NMC particles further deteriorate the level of CBD contrast.

In this study, for the first time this challenge has been addressed by a novel dual-scan superimposition technique called DSS to capture the full microstructure of the NMC electrode including the authentic CBD: a second X-ray nano-CT scan of the separate stand-alone CBD sample (i.e. prepared with no NMC particles) with a much higher voxel resolution $(63 \mathrm{~nm})$ was undertaken so that the micro-pores inside the blurry phase could be identified due to the enhanced signal-to-noise ratio and better spatial resolution and contrast (inset in Fig. 1e, detailed scanning parameters in Methods section). By retaining the NMC particles and macro-pores (labelled as blue in Fig. 1f) in the first scan and replacing the blurry mixed phase with the reconstructed second scan, the fully representative electrode microstructure is obtained (Fig. 1g), which corresponds well to the SEM image (Fig. 1d) in terms of porosity and pore size distribution.

Figure 2a shows the reconstructed cathode with the three phases represented by greyscale values that are proportional to their respective X-ray attenuation coefficients, based on which the three phases are segmented (Fig. 2b) and a heterogeneous pore phase is observed. Figure $2 \mathrm{c}$ displays the colour-coded spatial distribution of pore size, whose heterogeneity correlates to the simulated Li-ion diffusive flux (Fig. 2d, simulation details in Supplementary Eq. 14 and Supplementary Fig. 2): the heterogeneity of the pore size results in locally high flux (i.e. bright areas) and could lead to regions of higher ionic transport resistance and elevated temperature. Figure $2 \mathrm{e}$ identifies each individual NMC particle, with the shape and orientation visualised in Fig. 2f. The particle size as a function of distance from the separator is plotted in Fig. $2 \mathrm{~g}$, showing a uniform distribution of the particle size along the through-thickness direction (perpendicular to the current collector) and a wide scattering (between 2 and $15 \mu \mathrm{m}$ ) at each depth of the electrode. Figure $2 \mathrm{~h}$ shows that larger particles are more spherical than smaller ones. An extremely broad sphericity distribution is observed, calling into question the validity of cathode models based on ideal spherical particle geometry. It is worth mentioning that the sphericity of the active particles has been observed to decrease during long-term cycling as a consequence of phase transformation and stress accumulation ${ }^{27}$. The orientation distribution of these particles is random (Fig. 2i). The volumeaveraged tortuosity factor $\tau$, which is often used to describe the 
a

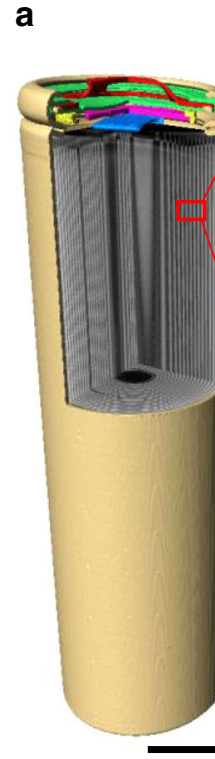

b

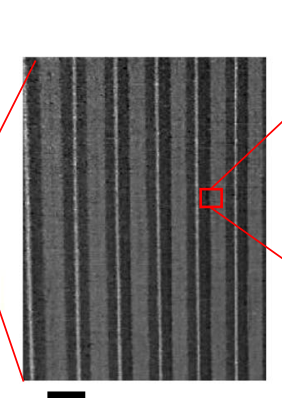

C

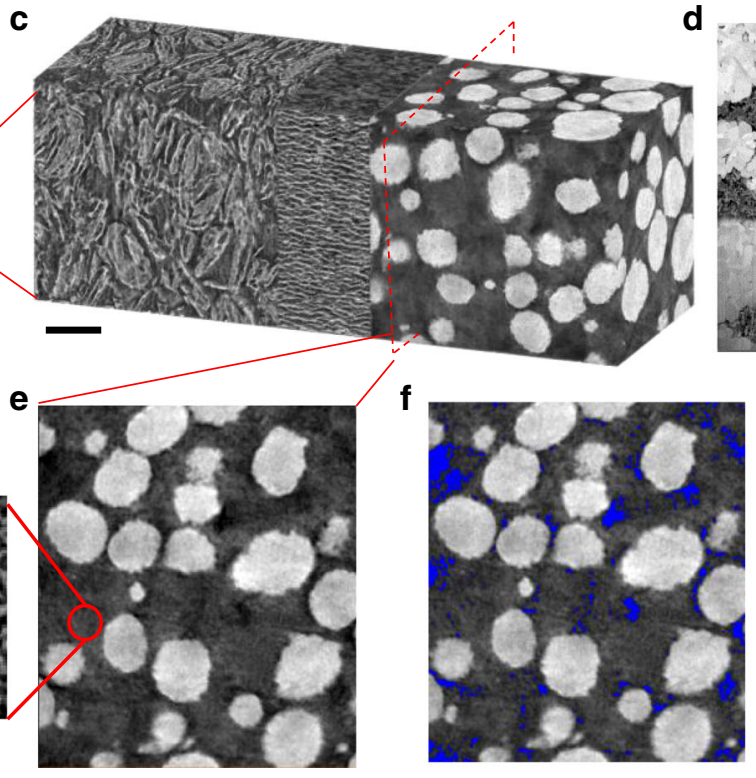

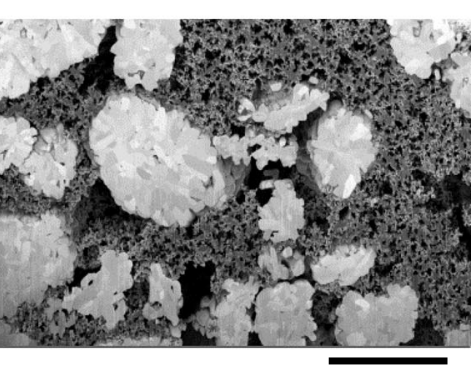

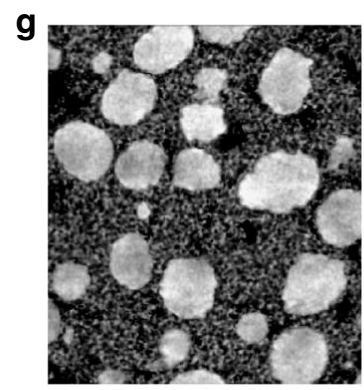

Fig. 1 Hierarchical structure of the LiB. a Volume rendering of the reconstructed cylindrical battery scanned by $\mathrm{X}$-ray micro-CT (accelerating voltage $180 \mathrm{kV}$, exposure time $1 \mathrm{~s}$ and voxel size $12.9 \mu \mathrm{m}$ ). The metal shell (brown), top button (red), venting disk (green), crimp plate (pink), seal insulator (yellow) and current collector (blue) are shown, with a corner cut displaying the internal structure of the battery; $\mathbf{b}$ magnified virtual slice to show the periodic layered structure of the cell; $\mathbf{c}$ X-ray nano-CT image showing (from left to right) the graphite anode, polyolefin separator and NMC cathode; $\mathbf{d}$ SEM image showing the CBD morphology alongside the secondary NMC particles, wherein the crystallography of the primary particles is seen; e virtual slice of the reconstructed 3D electrode, showing a blurry phase comprising the CBD and micro-pores (white: active material particles; dark grey: macro-pores; light grey: blurry mixed phase of micro-pores and CBD). Inset image shows the higher resolution scan of the CBD phase; $\mathbf{f}$ macro-pores (blue) are highlighted and retained with the active particle in the dual-scan superimposition process; $\mathbf{g}$ the resultant full microstructure of the NMC cathode by $\mathrm{X}$-ray $\mathrm{CT}$. The scale bars in (a, b) represent $10 \mathrm{~mm}$ and $240 \mu \mathrm{m}$ respectively, $10 \mu \mathrm{m}$ in $(\mathbf{c}, \mathbf{d}, \mathbf{e})$ and $2 \mu \mathrm{m}$ in the inset of (e).

effective conductivity/diffusivity of the pore phase, increases significantly over the first $30 \mu \mathrm{m}$ (four times the average particle size) from the starting plane (Fig. 2j), highlighting the inhomogeneous microstructure. The porosity displays severe fluctuation, which could arise from the local variation of the solid particle distribution (Fig. 2k), as supported by a good correspondence between the local porosity (blue curve in Fig. $2 \mathrm{k}$ ) and the particle distribution in Fig. $2 \mathrm{~g}$ (region $10-20 \mu \mathrm{m}$ from the separator). These are all microstructural heterogeneities that should be considered in electrode design, manufacturing and battery modelling. Figure 21 plots Bruggeman exponents ( $p$ in $\tau=$ $\phi^{-p}, \phi$ is the porosity) that increase from approx. 0.9 to 1.15 , significantly higher than the empirical value for spherical particles $(p=0.5)$.

Microstructure and performance correlation. To understand the interplay between battery microstructure and performance, dynamic discharging processes (i.e. cathode lithiation) at different C-rates were simulated using the reconstructed cathode microstructure and an ideal metallic lithium anode (Fig. 3). The illustration of the physical processes, mathematics and parameters is shown in Supplementary Fig. 4 and Supplementary Table 1-3. The simulated voltage vs. capacity curves are compared with the experimental data of the fabricated coin cells (NMC111 vs. lithium) at different C-rates (Supplementary Fig. 6). Figure 3 shows that the SoL is relatively uniform at $1.25 \mathrm{C}$, while a gradient starts to develop at $2.5 \mathrm{C}$ and becomes severe at $5 \mathrm{C}$. This is consistent with the increasing gradient along through-thickness direction of electrolyte salt concentration $C_{\text {ey }}$ in the second column. The competition between reaction kinetics and mass transport dynamics determines the SoL and $C_{\text {ey }}$ gradients: at high $\mathrm{C}$-rates the reaction is relatively fast so that the sluggish mass transport in the convoluted $3 \mathrm{D}$ pore phase cannot supply the reactant $\left(\mathrm{Li}^{+}\right.$ion) deep into the electrode at a sufficient rate. Consequently, the charge transfer predominantly occurs near the separator where $C_{\mathrm{ey}}$ is high, while the NMC particles that are further away are not fully utilised leading to severe capacity underutilisation. Furthermore, more homogeneous SoL of intraand inter-particles is observed at $1.25 \mathrm{C}$, compared to the higher C-rates wherein significant disparity of the SoL is evident between small and large particles and also from the core to the surface of the large particles, as a consequence of the ratio between the solid-state diffusion and reaction kinetics. The heterogeneous SoL inevitably creates temperature gradients and thermal/mechanical stress $^{28,29}$ both locally in individual particles and between neighbouring particles, leading to cracking and delamination that reduce the battery lifetime ${ }^{15}$.

The third column displays the distribution of $\mathrm{Li}^{+}$ion flux $J_{\mathrm{p}}$. At $1.25 \mathrm{C}$ the flux is evenly distributed; however, significant heterogeneity is observed beyond $2.5 \mathrm{C}$ due to the non-uniform pore microstructure, in which the $\mathrm{Li}^{+}$ion flux is much higher in narrower pores or in low porosity regions. This would cause local Ohmic heating, potentially giving rise to electrolyte decomposition $^{30}$ and initiating thermal runaway ${ }^{13}$. The final column shows the charge transfer $J_{c t}$ (i.e. reactivity) distribution at the electrolyte/ particle interface, which is observed to be more homogeneous at low C-rates whereas at high C-rates, reactivity variation is observed particle-by-particle as a consequence of local microstructural heterogeneity: regions with higher current densities are expected to degrade faster than average. In addition, due to the mass transport limitation, $J_{\mathrm{ct}}$ is significantly higher in the vicinity of the separator than the current collector at high C-rates, yielding the "switch-off" of substantial reaction active sites.

Analysis of the SoL in each particle in the through-thickness direction at different C-rates was conducted (Fig. 4). As the 

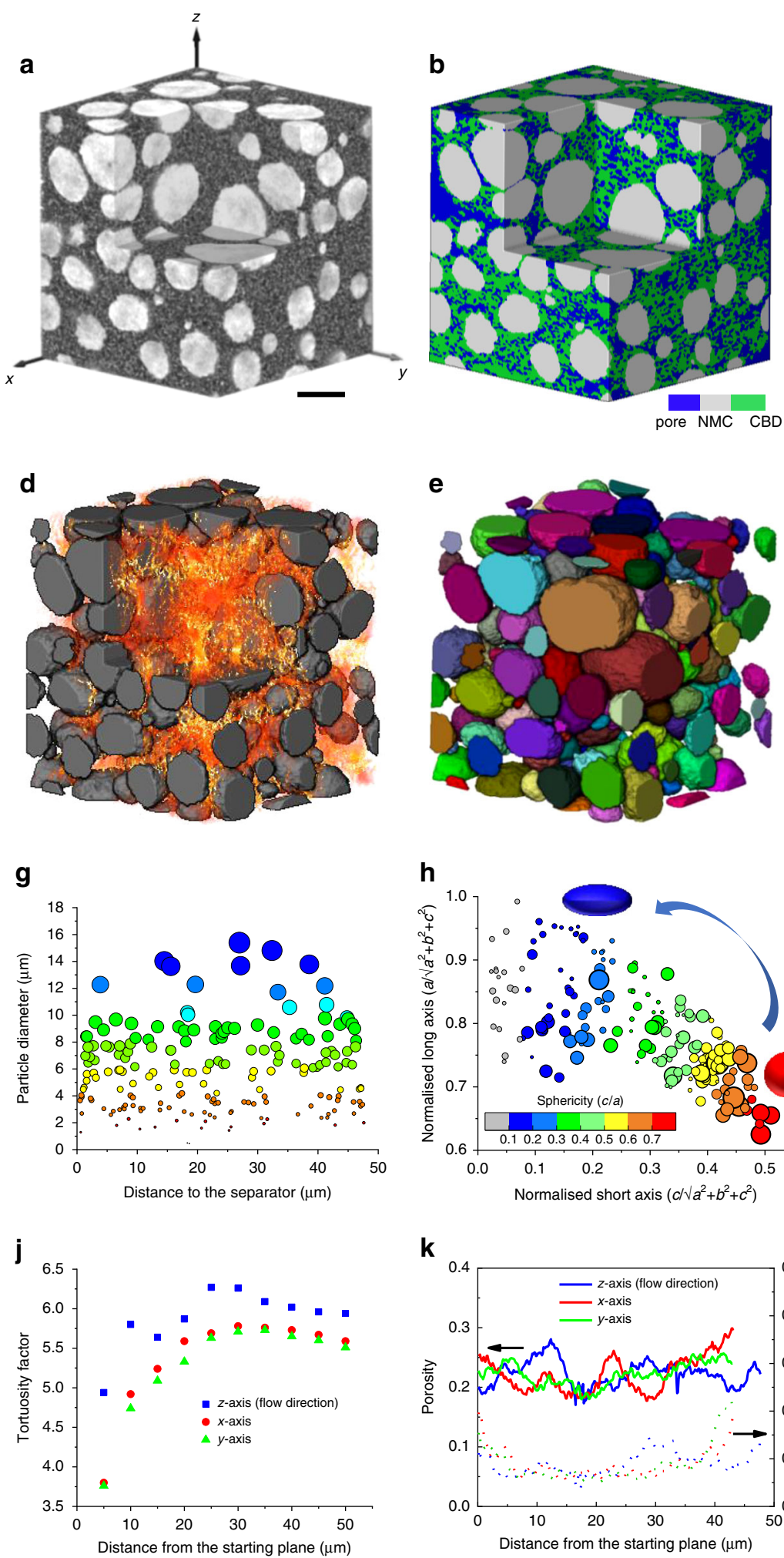
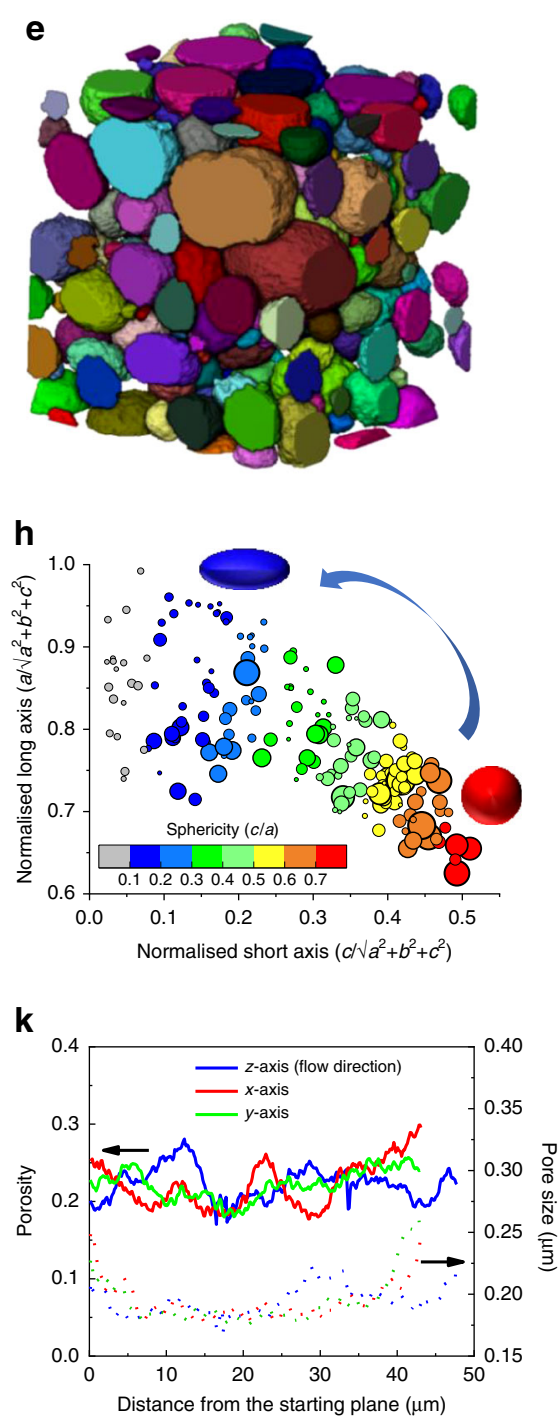
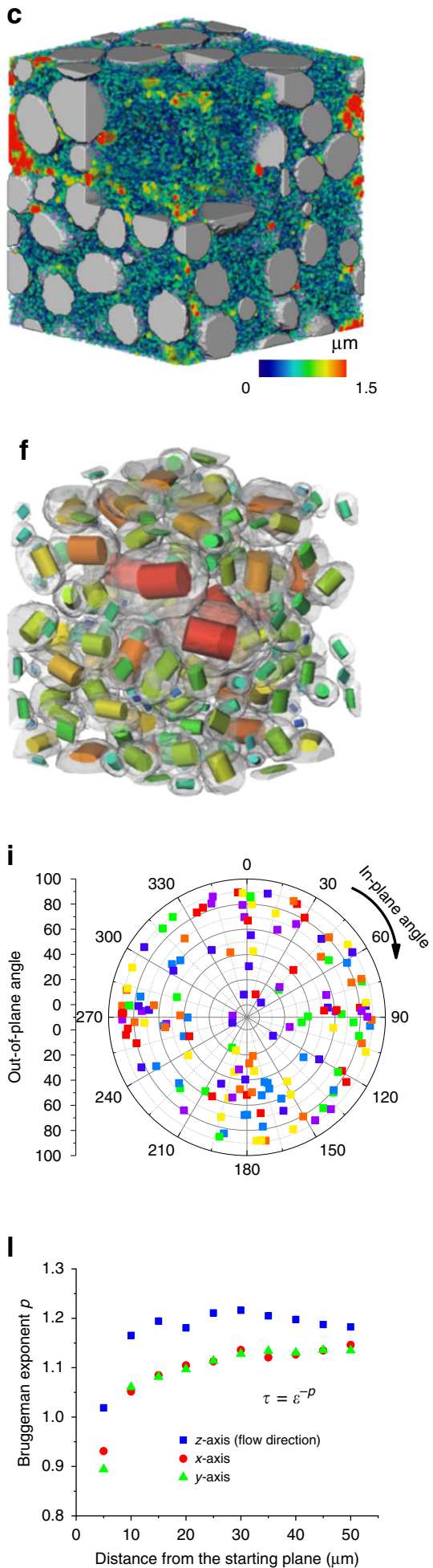

Fig. 2 3D characterisation of the NMC cathode based on X-ray CT data. a Reconstructed volume of the electrode with different phases represented by greyscale values (white: NMC active material; dark grey: CBD; black: pore). The corner is cut to show the internal morphology; b segmented three phases; c colour-coded pore phase according to its size, shown with NMC particles; $\mathbf{d} \mathrm{Li}+$ ion diffusive flux map in the pore phase used for effective mass transport estimation; e identification of individual NMC particles; $\mathbf{f}$ visualisation of the shape, orientation and size of each individual particle; $\mathbf{g}$ resultant particle size distribution as a function of distance from the separator. Symbols are colour-coded according to the particle size; $\mathbf{h}$ sphericity distribution of the NMC particles. The size of the scatter points represents the size of the particles and the colour codes represent the sphericities; $\mathbf{i}$ orientation map of the individual particles, with the azimuthal angles and radial distance representing the in-plane and out-of-plane orientations; $\mathbf{j}$ tortuosity factor obtained based on the diffusive flux from (d) in three directions; $\mathbf{k}$ slice-by-slice variation of porosity and pore size in three orthogonal directions; I Bruggeman exponents as a function of the fraction of the electrode thickness in three orthogonal directions. The scale bar in (a) represents $10 \mu \mathrm{m}$. 


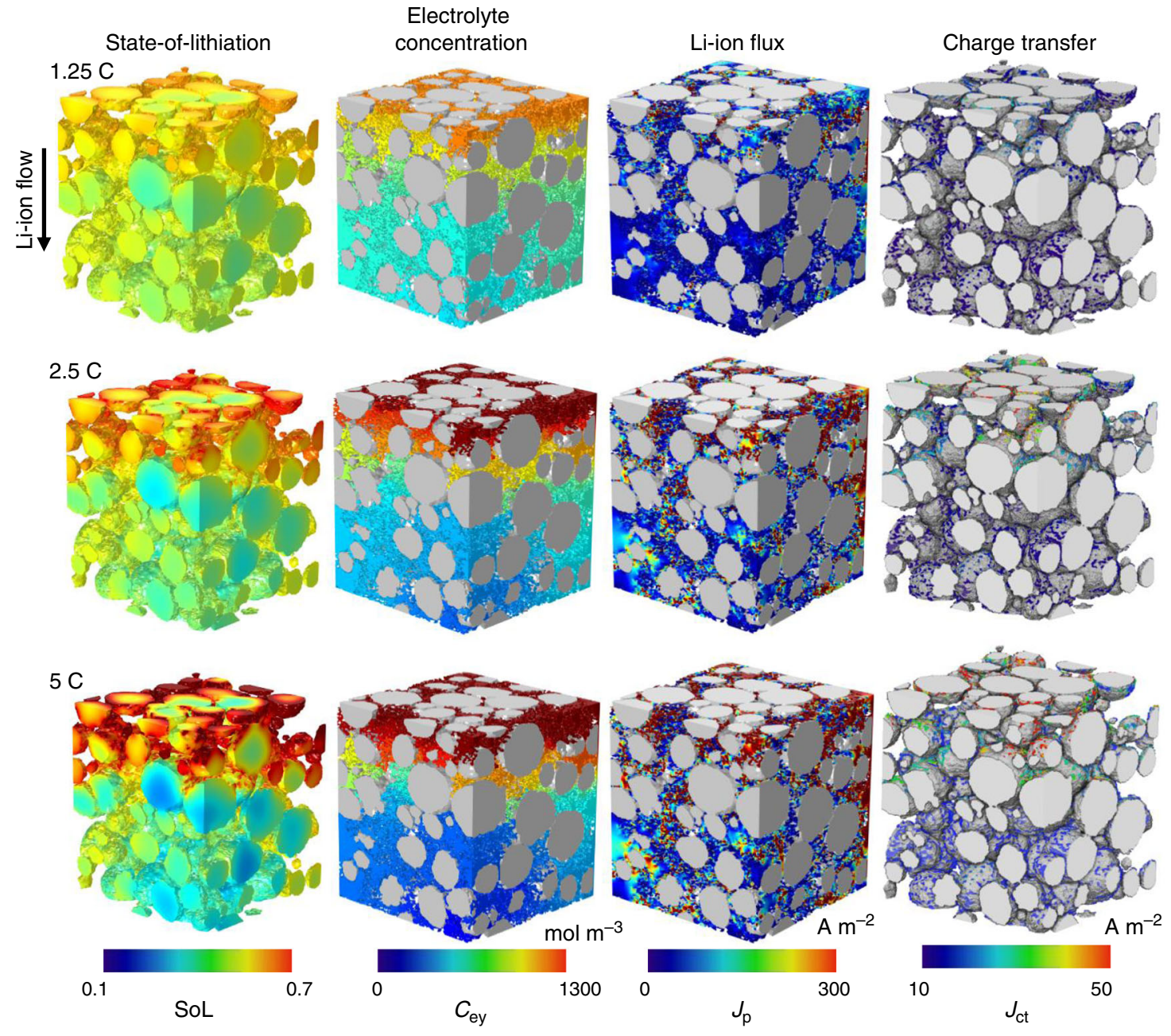

Fig. 3 Simulated discharge of the reconstructed cathode and an ideal lithium anode (half-cell) at 1.25, 2.5 and $5 \mathrm{C}$. The ionic current flows from the top (separator side) to the bottom (current collector side). Field variables at 50\% depth-of-discharge (DoD) are shown here.

discharge rate increases, the impact of particle size on SoL heterogeneity becomes more pronounced: at $5 \mathrm{C}$ significant heterogeneity is observed both transversely and longitudinally along the through-thickness direction, with up to $60 \%$ variation in SoL between small and large particles close to the separator (Fig. 4c). The insets in Fig. 4a-c compare the uniformity of lithium concentration at three discharge rates at $50 \%$ depth-ofdischarge (DoD). The histogram in Fig. 4d shows the macroscopically narrow distribution of SoL at $50 \% \mathrm{DoD}, 1.25 \mathrm{C}$, with two distinct peaks, representing a more uniform particle lithiation compared to Fig. 4e, which displays a broader SoL distribution at $5 \mathrm{C}$. The three distinguishable peaks are more separated (i.e. incrementally severe lithiation separation) as a function of discharge time (Supplementary Fig. 7), which is consistent with the electrochemical Biot number plot (Supplementary Fig. 8) that characterises the relative rates between solid-state diffusion and interfacial reaction kinetics. The inset in Fig. $4 \mathrm{e}$ visualises the particles corresponding to the three peak regimes and correlates SoL with particle size and depth. Figure $4 \mathrm{f}$ compares the SoL at the three discharge rates, highlighting how the heterogeneous SoL builds up locally and along the through-thickness direction as Crate increases. In summary, the inhomogeneous lithiation is mainly due to the variation in lithium ion diffusion path associated with the broad distribution of particle size. The uneven utilisation of the NMC particles inevitably leads to a lower energy density than the theoretical one, and likely undermines the long-term structural integrity of the electrode.

The heterogeneous SoL is also a consequence of non-uniform current density distribution. Figure $5 \mathrm{a}$ exhibits the distribution of charge transfer current density $J_{\mathrm{ct}}$ at the NMC particle/electrolyte interface at $5 \mathrm{C}$. The spatial distribution of exchange current density $j_{0}$ and activation overpotential $\eta_{\text {act }}$ are displayed in Fig. $5 \mathrm{~b}$ and $c$ respectively, which are linked to $J_{\mathrm{ct}}$ via the Butler-Volmer equation (Supplementary Eq. 11). The $\eta_{\text {act }}$ is in good agreement with the experimentally measured values ${ }^{31}$. $2 \mathrm{D}$ histogram correlation is used to analyse the spatial co-localisation of $J_{\mathrm{ct}}$ with $j_{0}$ and $\eta_{\text {act }}$ respectively, concluding that the non-uniform $J_{\mathrm{ct}}$ is strongly correlated with $\eta_{\text {act }}$ rather than $j_{0}$ (Supplementary Fig. 9). This is reasonable considering that $J_{\mathrm{ct}}$ is exponentially dependent on $\eta_{\text {act }}$, which is further decomposed to equilibrium potential $V_{\text {eq }}$ and electrolyte potential $\varphi_{\mathrm{p}}$ at the reaction interface (Supplementary Fig. $9 \mathrm{e}-\mathrm{f}$ ). In view of the larger local variation of $\varphi_{\mathrm{p}}\left(\Delta \varphi_{\mathrm{p}}=0.080 \mathrm{~V}\right)$ compared to $V_{\mathrm{eq}}\left(\Delta V_{\mathrm{eq}}=0.025 \mathrm{~V}\right)$, which is essentially dependent on SoL, we conclude that $\eta_{\text {act }}$ is mainly governed by $\varphi_{\mathrm{p}}$ (more discussion in Supplementary Note 5). Thus, it is necessary to determine the cause of heterogeneity in $\varphi_{\mathrm{p}}$ to understand the non-uniform current distribution.

Since Ohm's law governs ion migration in the electrolyte ${ }^{32}$, the local value of $\varphi_{\mathrm{p}}$ is determined by the potential drop in the electrolyte, associated with transport distance from the separator 

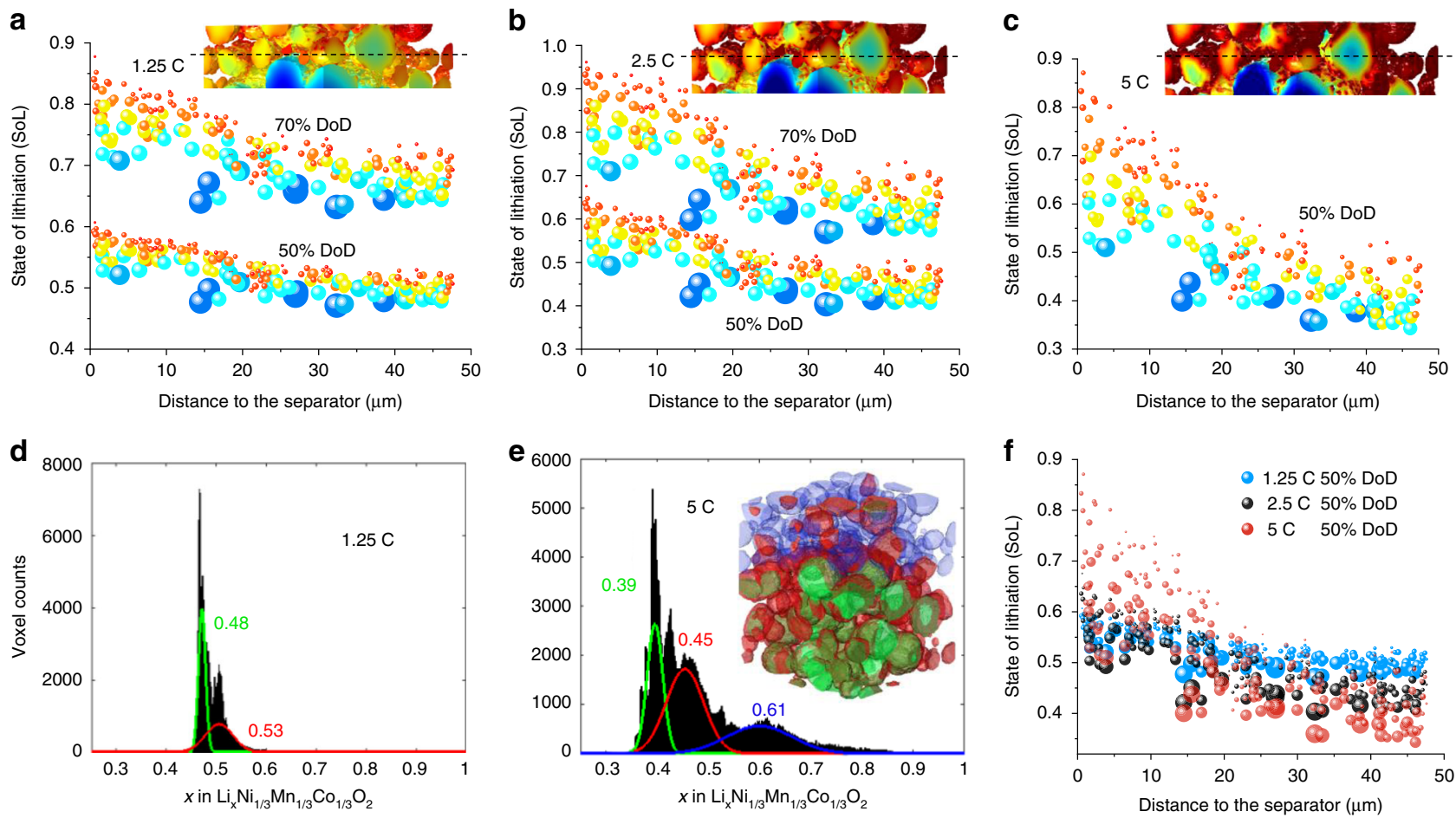

Fig. 4 Analysis of particle-by-particle variation of SoL under different operating conditions. The colour of the symbols corresponds to the particle size (blue to red: large to small) to enhance the visibility, and size of the symbols is proportional to the particle size. At 5 C, 70\% DoD is not accessible so only $50 \%$ is shown. The insets in (a-c) compare the different degrees of heterogeneous SoL in the same region ( $15 \mu \mathrm{m}$ from the separator) as a function of discharge rate. $\mathbf{d}$ and $\mathbf{e}$ compare the histogram of SoL in the active material at discharge rates of 1.25 and $5 \mathrm{C}$ where the SoL values of main peaks are identified with different colours. The particles in the 3D rendering in (e) are colour-coded according to the three peaks in the histogram, and $\mathbf{f}$ comparison of SoL of the corresponding individual particles at three discharge rates.

(Fig. 5d). Although the electrode thickness is $50 \mu \mathrm{m}$, the actual travel distance for the pore close to the current collector is more than twice this value, implying a substantial impact of convoluted $3 \mathrm{D}$ trajectory on mass transport. A magnified region from Fig. $5 \mathrm{~d}$ shows the local variation of travel distance of lithium ions to the interfacial reacting site (Fig. 5e), which turns out to be inversely correlated with the potential distribution in the electrolyte $\varphi_{\mathrm{p}}$ (Fig. 5f), consistent with the heterogeneous charge transfer current density (Fig. 5g). Therefore, we can attribute the local variation of reaction activity to the microstructural heterogeneity which causes the local discrepancy of $\varphi_{\mathrm{p}}$. This is further supported by analysis of local tortuosity at each individual reaction site, which can provide more geometrical insights than the widely used macro-homogeneous tortuosity factor ${ }^{33,34}$. Two types of tortuosity are characterised (Fig. $5 \mathrm{~h}$ ): (1) $\tau_{\text {pore }}$, the ratio of the shortest travel distance (blue trajectory) to the Euclidean distance (red dashed line) from the top plane to the reaction site, representing the resistance associated exclusively with the pore phase; (2) $\tau_{\text {electrode, }}$ calculated by dividing the shortest travel distance by the vertical distance (green dashed line) to the top plane, delineating the tortuous pathway as a result of both the pore phase and particle morphology.

Figure $5 \mathrm{i}$ plots tortuosity vs. distance from the separator for all reaction sites. There is a sharp decrease in $\tau_{\text {electrode }}$ within $15 \mu \mathrm{m}$ of the separator/electrode interface, with a large scatter followed by a plateau in which $\tau_{\text {electrode }}$ converges to $3 . \tau_{\text {pore }}$ exhibits a lower gradient than $\tau_{\text {electrode }}$ close to the separator and converges to the same level as $\tau_{\text {electrode }}$ after $15 \mu \mathrm{m}$. This further emphasises the contribution of particle size and shape to the transport resistance of lithium ions close to the separator, where the particle morphology dominates the ion transport path. As the distance from the separator increases, the impact of particle size and elongation decreases, thus $\tau_{\text {electrode }}$ and $\tau_{\text {pore }}$ reach similar values close to the current collector. This also implies that, at low C-rates when the battery performance is not mass-transport limited, a volume-averaged tortuosity factor can be safely used for model prediction. However, at high C-rates, the performance is heavily dependent on mass transport properties which are primarily determined by particle size and shape close to the separator. Under these circumstances, using a volume-averaged tortuosity factor will inevitably lead to an over-estimation of the lithium-ion flux which can propagate into the parameterisation process for battery management systems (BMSs).

Design and optimisation of next-generation electrodes. The above insights can be applied to the design of improved gradedmicrostructure electrodes, which have recently attracted increasing attention 35,36 . Compared with the as-prepared microstructure (Fig. 6a), here we obtain the graded-particle structure (Fig. 6b) by morphological erosion 37,38 of the initial particles in a region within $12 \mu \mathrm{m}$ of the separator/electrode interface and filling the gap between particles with small ones so as to maintain the same active material volume fraction (see Methods for more details). The decrease of particle size benefits the electrochemical performance by increasing the number of interfacial reaction sites (Fig. 6c), thereby enhancing the surface exchange kinetics. The electrochemical performance is compared at $50 \%$ DoD and $3.75 \mathrm{C}$ discharge. The second column in Fig. 6 shows that in the fineparticle zone the electrolyte distribution is more uniform, associated with the reduced $\mathrm{Li}^{+}$ion transport resistance due to the particle geometry. The third column evidences a significant homogenous $J_{c t}$ distribution in the graded-particle sample (Fig. 6h) compared to the as-prepared one (Fig. 6g), which arises 


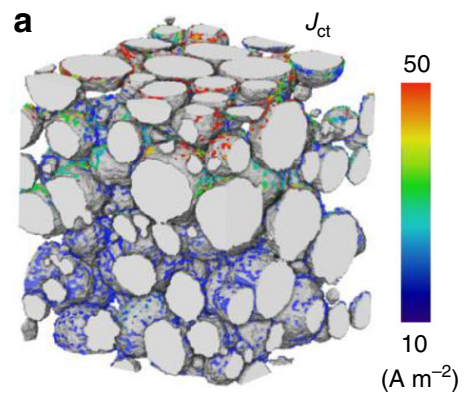

d
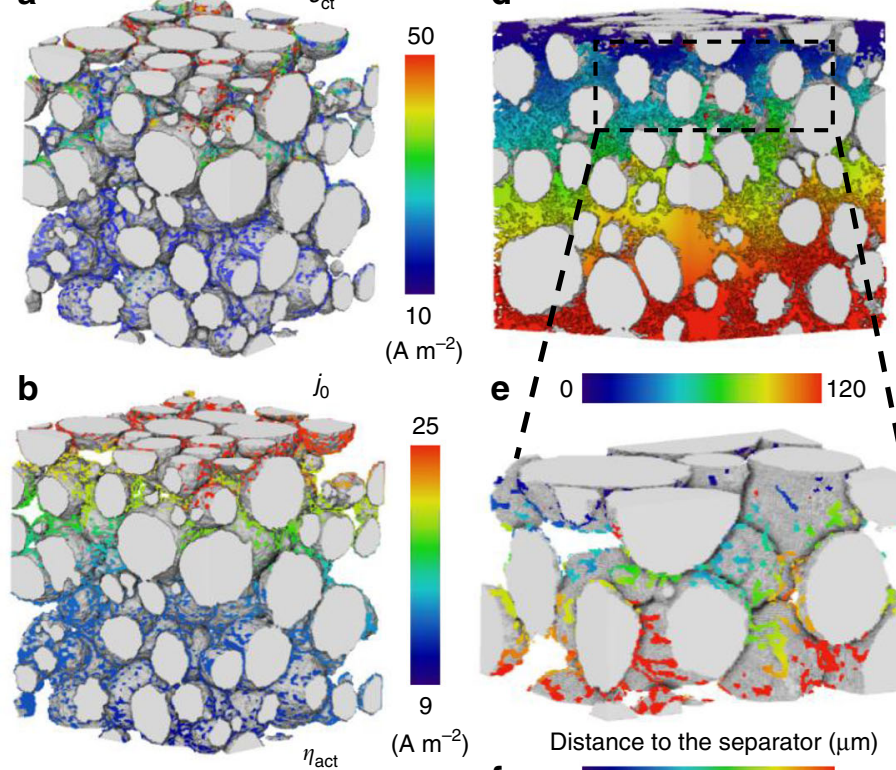

C

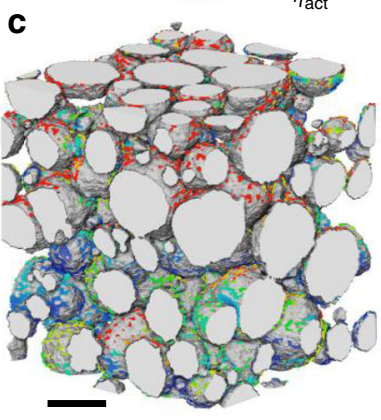

$\left(A \mathrm{~m}^{-2}\right)$

\section{e I}

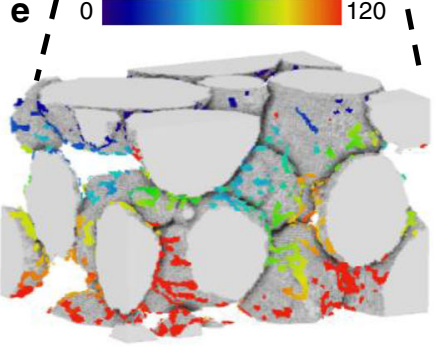

Distance to the separator $(\mu \mathrm{m})$

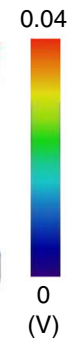

f

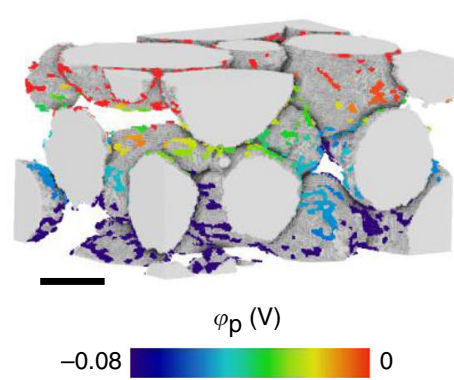

g

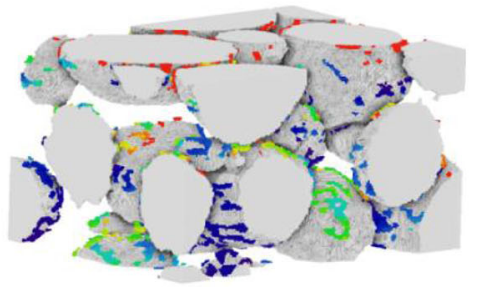

h $\quad 15 \square$

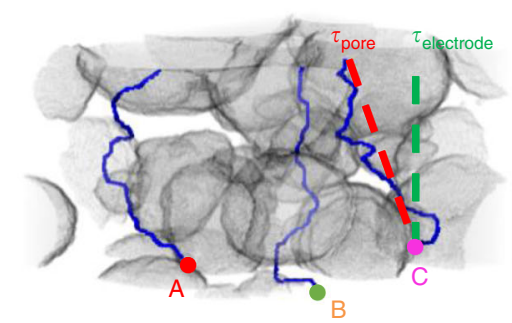

i

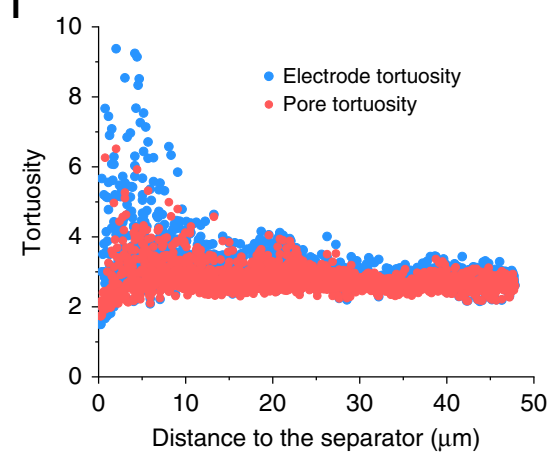

Fig. 5 Heterogeneous charge transfer analysis. Spatial distribution of a charge transfer current density $J_{\text {ct }} ; \mathbf{b}$ exchange current density $j_{0} ; \mathbf{c}$ activation overpotential $\eta_{\text {act }} ; \mathbf{d}$ distance map of every single voxel in the pore phase to the nearest electrode/separator interface measured by a 3D fast marching algorithm; e travel distance of the lithium ion to the reaction sites is well correlated with $\mathbf{f}$ the potential drop in the electrolyte $\left(\varphi_{\mathrm{p}}\right)$, which is consistent with $\mathbf{g}$ the local variation of charge transfer current density $J_{\text {ct. }} \mathbf{h}$ Illustration of the tortuosity in the pore phase $\tau_{\text {pore }}$ and electrode $\tau_{\text {electrode }}$ respectively. A, B and $\mathrm{C}$ exemplify three distinctly convoluted pathways for $\mathrm{Li}^{+}$ion flow; $\mathbf{i}$ plot of $\tau_{\text {electrode }}$ and $\tau_{\text {pore }}$ as a function of distance from the separator. The scale bars in (c) and (f) represent 10 and $7 \mu \mathrm{m}$, respectively.

from the higher specific surface area and more uniform potential distribution in the electrolyte, contributing to an increased discharge capacity and average voltage (Fig. 6i). The graded-particle structure provides more homogeneous lithiation and better utilisation of active material (Fig. 6k vs. Fig. 6j and Fig. 6l).

The electrode electrochemical performance is closely related to mass transport capability, especially close to the separator for high C-rates. To investigate this, morphological dilation ${ }^{37,38}$ of the local pore structure (see Methods for more details) was conducted to enlarge the pore size by $250 \mathrm{~nm}$ at three distances away from the separator to obtain graded-pore structures (R1, R2 and R3 in Fig. $7 \mathrm{a}-\mathrm{c}$ ). The porosity increases by shrinking the $\mathrm{CBD}$, while the NMC particles are unchanged. In Fig. 7j, the black curve represents the original porosity as a function of the depth in the as-prepared sample, while the porosity in the modified regions is labelled as R1, R2 and R3. In this way, the contribution of the pores to the global electrochemical performance can be decoupled from the particle microstructure, which is not possible experimentally due to sample-to-sample variations.

Figure $7 \mathrm{~d}-\mathrm{f}$ compare the SoL between the three samples at the end of discharge process at $3.75 \mathrm{C}$. Sample R1 significantly outperforms the other two. Combined with Fig. 7k, each sample shows respectively the lowest gradient of electrolyte concentration in the corresponding processed region (Fig. $7 \mathrm{~g}-\mathrm{i}$ ), due to the locally enhanced mass transport, as the graded-particle electrode demonstrated previously. Although Samples R2 and R3 exhibit similar performance, the locally boosted mass transport still yields a better performance compared to the as-prepared electrode (Fig. 7l). Sample R1 shows a considerable increase in the accessible capacity. It is noted that the rise of local porosity not only enhances the mass transport but is also accompanied by an increase of active particle/electrolyte interface. As the C-rate increases, the reaction active region resides closer to the separator, where R1 provides the most electrochemically favourable conditions compared to R2 and R3. A high porosity sample is also added for comparison (i.e. $\mathrm{R} 1+\mathrm{R} 2+\mathrm{R} 3+\mathrm{R} 4$, porosity approx. $33 \%$ ), displaying $16 \%$ more capacity than R1 with a similar average voltage. These results indicate that the battery electrochemical performance is predominantly governed by the porosity at the separator/electrode interface at high C-rates, proving the efficacy of graded-structure electrode design.

The performance of each electrode design is summarised using the Ragone plot in Fig. 8a. Regarding the as-prepared electrode as a baseline, there is great possibility to improve the battery performance using different strategies. In this study the increase in porosity was achieved by pushing the pore/CBD boundary 
a

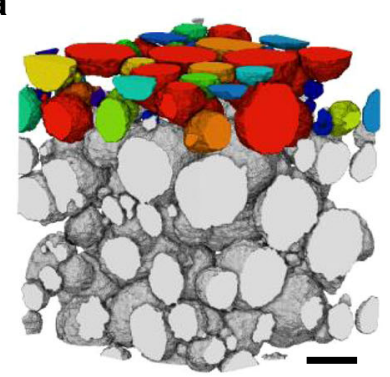

b

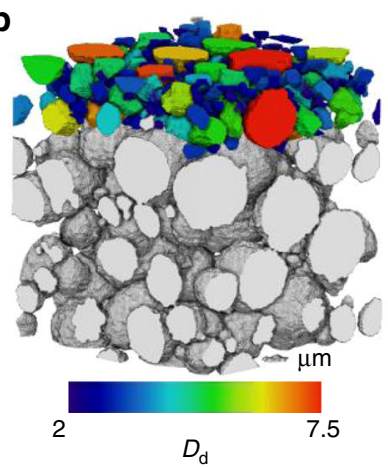

C

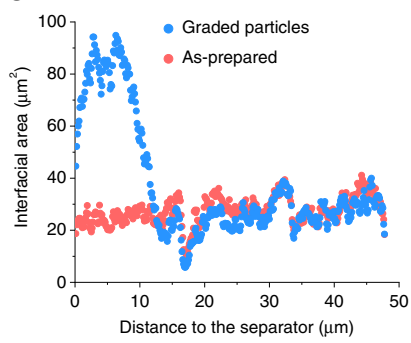

d

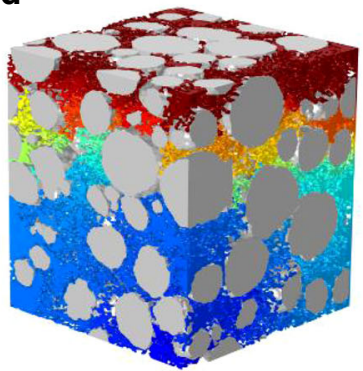

e

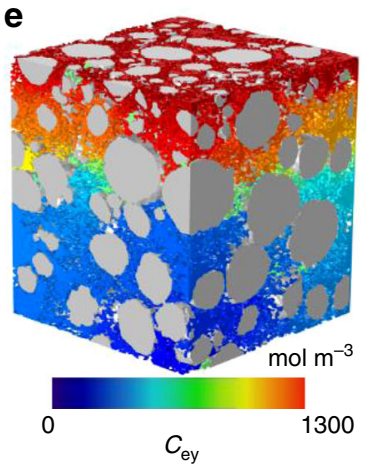

f

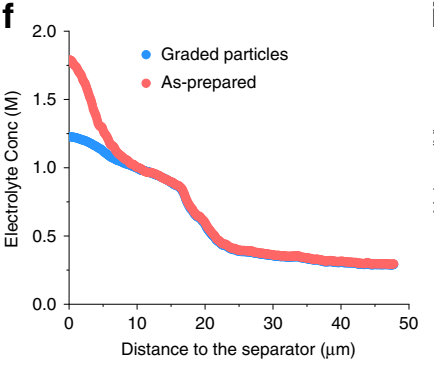

g

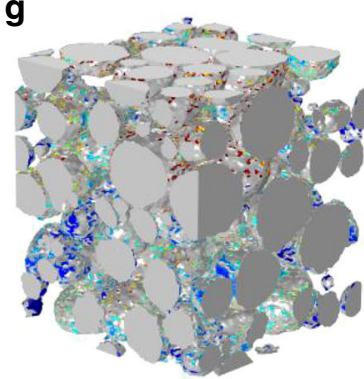

h

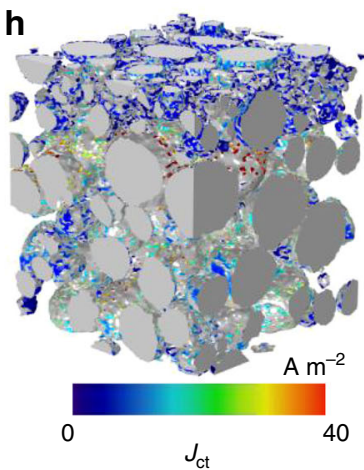

i

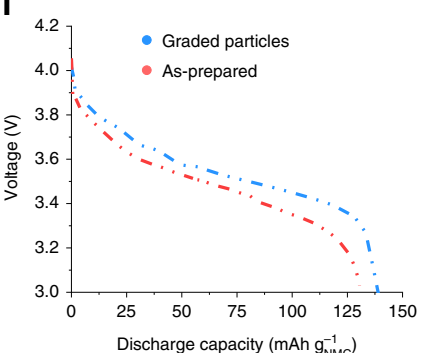

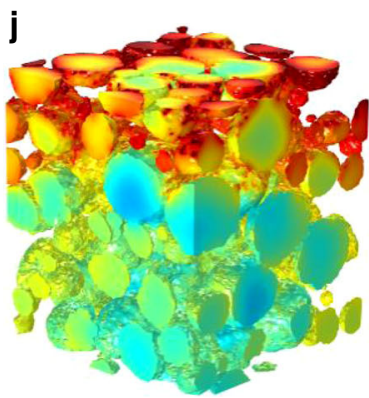

k
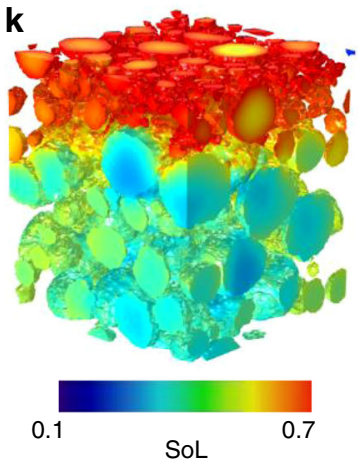

SoL

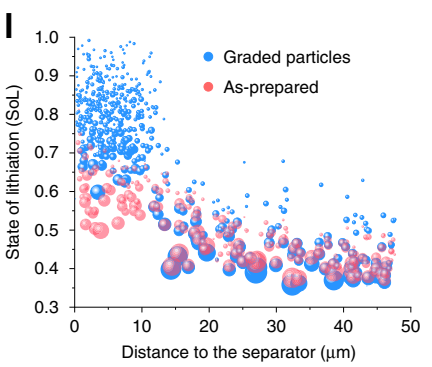

Fig. 6 Comparison between the as-prepared (top row) and graded-particle (middle row) samples at 50\% DoD and 3.75 C discharge. a, b Comparison of the particle size distribution; c change in interfacial reaction area as a function of the depth in the electrode; $\mathbf{d}-\mathbf{f}$ comparison of the distribution of the electrolyte concentration; $\mathbf{g}, \mathbf{h}$ distribution of charge transfer current density and $\mathbf{i}$ the resultant voltage vs. capacity curve; $\mathbf{j}-\mathbf{I}$ comparisons of the SoL. The scale bar in (a) represents $10 \mu \mathrm{m}$.

towards the $\mathrm{CBD}$ while maintaining the voxels constituting the active material unchanged so that the NMC mass loading is constant. At the porosity level studied, no obvious polarisation arose from the electron-conducting phase (CBD and NMC); however, with further increase in porosity, the connectivity of electron-conducting pathways could be reduced, undermining the accessible specific energy, which will be investigated further in the next section. Thus, a homogeneous distribution of the solid materials is critical in pursuing high porosity microstructures.

For LiBs designed for hybrid applications, the graded-pore structure (light blue curve in Fig. 8) will be a prospective choice for microstructural optimisation as it provides considerable enhancement of both specific energy and power. This structure can be achieved by the addition of pore-formers or other advanced techniques such as ice-templating ${ }^{39,40}$. Since the porosity in the vicinity of the current collector is not very important at high C-rates, it is advised to maintain a high mass loading in this region instead of sacrificing volumetric energy density available at low C-rates for only a modest increase in power density at high C-rates.

For applications prioritising the maximum stored energy, large area-specific mass loading $\left(\mathrm{g} \mathrm{cm}^{-2}\right)$ is the most common choice. In Fig. 8a, a cathode of $75 \mu \mathrm{m}$ thickness (green curve) suffers from loss of power density. In addition, the thicker electrode shows inferior specific energy, deteriorating significantly with the increase of electrical load, as also proved by experimental data ${ }^{41}$. Given the trade-off between specific energy and power, a critical "knee point" should be noted on each curve (dashed red arrow). This point represents the highest accessible specific power before a catastrophic drop of specific energy, which is often integrated in BMSs to maximise the energy output while ensuring battery durability. Apart from the presented designs, extended studies can be conducted to examine the influence of materials processing on the specific power and energy.

Figure $8 \mathrm{~b}$ compares the specific accessible capacity of these designs as a function of the C-rate. All samples manifest almost identical accessible specific capacity at very low C-rate $(0.125 \mathrm{C})$, but the capacity falls in a distinct manner with increasing $\mathrm{C}$-rate as a consequence of the increasing polarisation of reactant transport and reaction kinetics. Both of the graded-structures show substantial improvement compared to the as-prepared electrode (grey curve), especially at higher C-rates. The spatial distribution of SoL for the as-prepared (Volume 1), high porosity (Volumes 2 and 3) and thick electrodes (Volume 4) at 60\% DoD is presented alongside the discharge curves. Comparing Volumes 1 and 2, the enlarged pore size assists a more uniform lithiation along the through-thickness direction; however, intra-particle heterogeneity still exists even in the high porosity sample, 

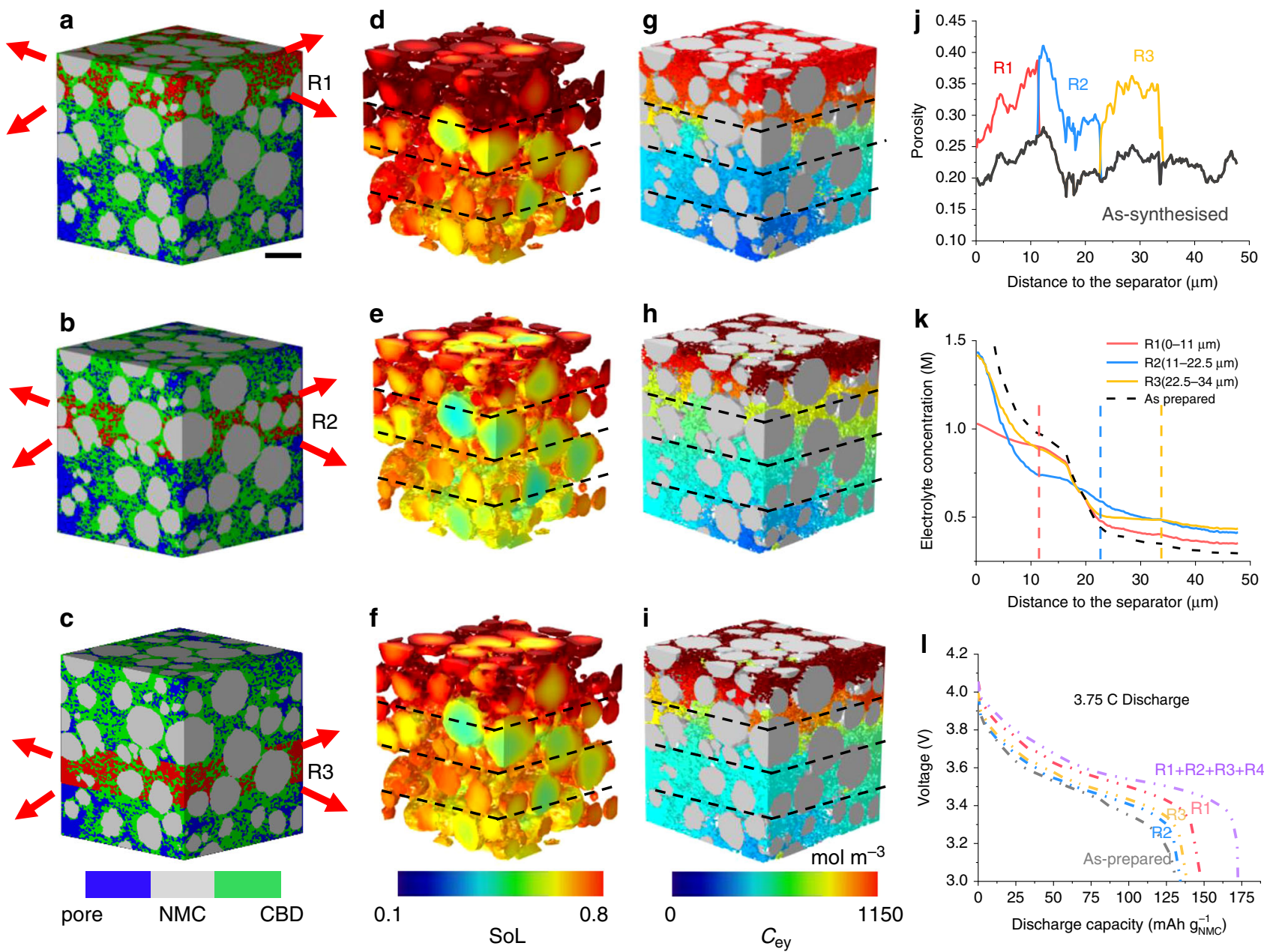

Fig. 7 Investigation of LiB electrode design with graded-porosity/pore size. Pore microstructure is enlarged locally by $250 \mathrm{~nm}$ : a $11 \mu \mathrm{m}$ (R1), b $22.5 \mu \mathrm{m}$ (R2), and $\mathbf{c} 34 \mu \mathrm{m}$ (R3) away from the separator. The processed regions are highlighted in red; $\mathbf{d}$-f maximum accessible SoL comparison between the three samples ( $84 \%, 77 \%$ and $76 \% \mathrm{DoD})$ discharged at $3.75 \mathrm{C}$, with the dashed lines (also those in (k)) representing the boundaries of the three processed zones; $\mathbf{g}-\mathbf{i}$ the corresponding distribution of electrolyte concentration; $\mathbf{j}$ plot of porosity vs. electrode thickness for the three samples, labelled as R1, R2 and R3, with the grey curve representing the baseline for the as-prepared sample; $\mathbf{k}$ local concentration gradient suppressed by the enlarged pores in each region at 50\% DoD; I comparison of electrochemical performance for different microstructural designs. R1 + R2 + R3 + R4 refers to global dilation of the pore microstructure. The scale bar in (a) represents $10 \mu \mathrm{m}$.

implying a limiting effect of solid-state diffusion. Volume 3 exhibits a more severe intra- and inter-particle difference of SoL due to the exacerbated competition between solid-state diffusion and surface exchange rate at $10 \mathrm{C}$ compared to Volume $2(3.75 \mathrm{C}$, the onset of $\mathrm{Li}^{+}$ion transport limitation). Volume 4 also shows a wide distribution of $\mathrm{SoL}$ even at $1.25 \mathrm{C}$, highlighting the predominant resistance from mass transport, which may be reduced by enhancing the cationic transference number in the electrolyte ${ }^{24}$.

So far in this study, the influence of microstructural heterogeneity (particle and pore size distribution, spatial arrangements and local mass transport property) on the energy and power density, the efficacy of graded-structure and a comprehensive comparison of different electrode microstructures have been investigated. The image-based modelling technique has been shown as a promising tool for advanced microstructural design and optimisation. To better convert the improved knowledge acquired in the section above to battery engineering, the next section will introduce the application of image-based modelling and DSS technique in rationalising the manufacturing protocols for desired microstructures.
Time-lapse X-ray nano-CT to track microstructural evolution during calendering. A time-lapse X-ray nano-CT experiment was conducted to track the microstructural evolution of the electrode under incremental calendering steps. A novel nanomechanical test stage was developed for integration into the Zeiss Xradia Ultra series X-ray microscopes ${ }^{42}$. The assembly and experimental setup are shown in Fig. 9a while Fig. 9b shows the alignment of the electrode pillar $(65 \mu \mathrm{m})$ under the compression head. Figure $9 \mathrm{c}$ is an X-ray radiographic projection showing the positioning of each component. Tomographic slices of three different compression steps are displayed in Fig. 9d-f. From compressive strain level $\varepsilon=7 \%$ to $\varepsilon=15 \%$, an NMC particle is observed to roll against the adjacent ones (yellow arrows). Both the macro and micro-pores decrease in size. With the increase of compression $(\varepsilon=22.5 \%)$, apart from the further decrease in porosity and pore size, the free-moving particle is constrained by the surroundings and deforms significantly in the horizontal direction, which results in a more compacted structure (red arrows). Figure $9 \mathrm{~g}-\mathrm{i}$ displays the 2D-projected mass flux map from the image-based computed fluid dynamics (CFD) simulation on the calendered 3D pore structure at respective 

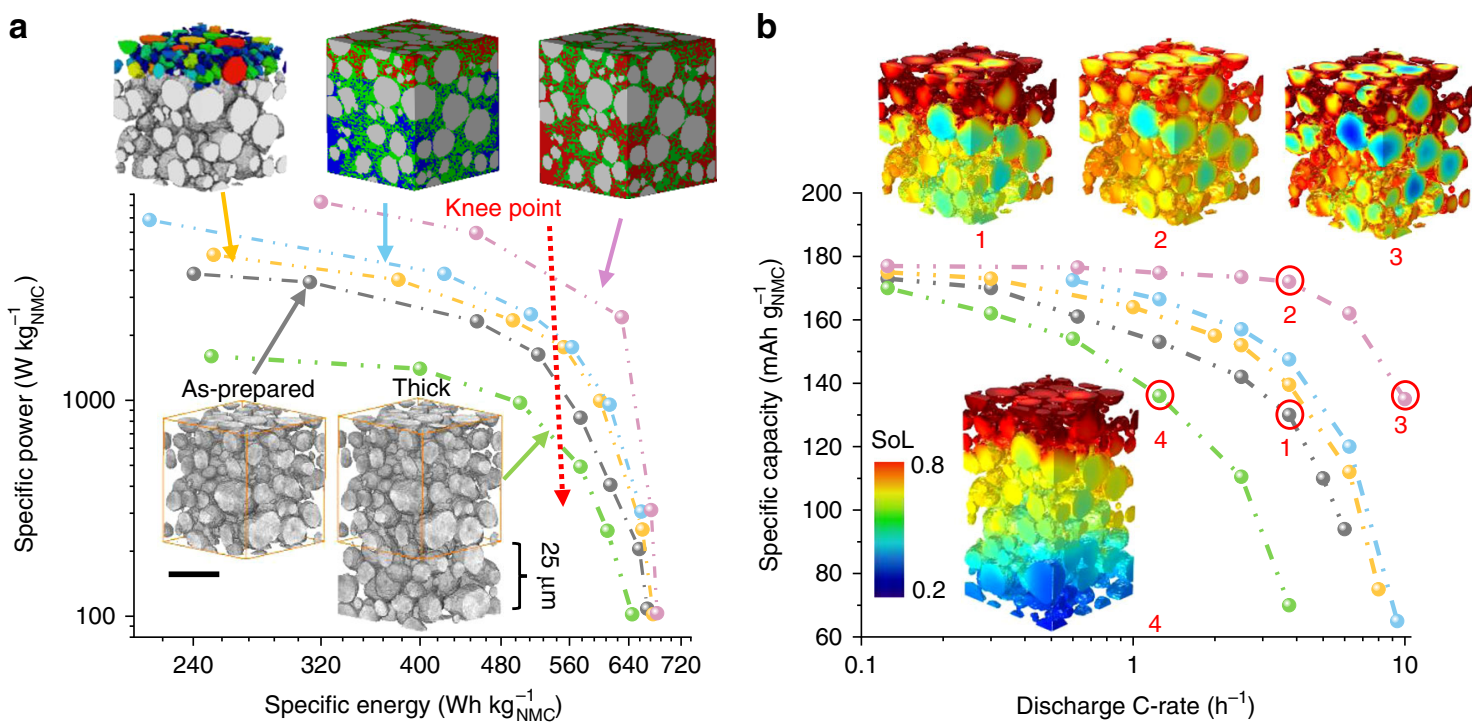

Fig. 8 Comparison of the simulated electrochemical performance for different electrode designs. a Ragone plot comparing the specific power vs. energy for a variety of electrode microstructures in lithium metal half-cell (grey: $50 \mu \mathrm{m}$ electrode; green: $75 \mu \mathrm{m}$ electrode; yellow: graded particle; light blue: graded pore; pink: high porosity). The specific energy is obtained by integrating the discharge curve to the cut-off voltage ( $3 \mathrm{~V})$ and the specific power is obtained by dividing the specific energy with the total discharge time; $\mathbf{b}$ maximum accessible specific capacity as a function of $\mathrm{C}$-rate for different microstructures. The inset figures compare the spatial distribution of SoL under different conditions, corresponding to the numbers on the curve respectively. The scale bar in (a) represents $20 \mu \mathrm{m}$.

compression levels, with the colour legend overlaid by the histogram. It is noted that the global intensity of the mass flux drops as a consequence of the loss of porosity. The localised high flux region (yellow) diminishes as well, reflecting the closure of narrow pores. It is concluded that when the calendering strain is above $20 \%$, the electrode becomes more compacted and significant loss of porosity could be found locally, either close to the current collector (white arrow in Fig. 9i) or in the vicinity of the active particles (black arrow). Figure $9 \mathrm{j}$ and $\mathrm{k}$ summarises the variation of the pore size distribution and the structural parameters of the electrode under the incremental calendering steps, which can not only be used to predict the tortuosity based on porosity and therefore guide electrode manufacture, but is also regarded as a reliable parameterisation to be used in reducedorder models for BMSs. The inset in Fig. 9j displays the 'crushed' $\mathrm{CBD}$ between adjacent particles and the consequent loss of reaction sites.

Additional insights into electrode engineering come from the analysis of how the same calendering strain affects electrodes with different mass loadings. Three mass loading electrodes $(1,2$ and $3 \mathrm{mAh} \mathrm{cm}^{-2}$ ) were prepared both uncalendered $(30,50,85 \pm 0.8$ $\mu \mathrm{m})$ and calendered at $20 \%$ compression $(24,40,68 \pm 0.6 \mu \mathrm{m})$ which were subsequently electrochemically cycled at different $\mathrm{C}$ rates (Fig. 9i). Three repeated tests were carried out on each type of the electrodes. The same calendering strain affected the electrodes with different mass loadings distinctly: for the low mass loading electrode higher specific capacity was obtained without calendering, while the opposite applied to the high mass loading electrode (Fig. 9i). These opposite trends are rationalised by the insights gained in the previous sections on electrode heterogeneity: (1) in the low mass loading electrode, calendering exacerbates the microstructural heterogeneity as compression can 'switch-off a substantial number of electrochemical-reactive sites due to the porosity decrease (red arrows in Supplementary Fig. 10), leading to capacity loss regardless of the C-rate. This suggests that the particle size used $\left(D_{50} \approx 5 \mu \mathrm{m}\right.$ and $D_{90} \approx 10 \mu \mathrm{m}$; see Supplementary Fig. 11) is too large to provide sufficient specific and total NMC particle/electrolyte interfacial area, consequently incurring inhomogeneous reaction and capacity underutilisation. The general trend of rate capability, though, is identical in the calendered and uncalendered electrodes, indicating that $\mathrm{Li}^{+}$ion transport is not rate-limiting; (2) the calendered high mass loading electrode performs better than the uncalendered one at low C-rate, while almost identical specific capacity is obtained at high C-rate. This is attributed to the long-range electrical percolation and contact that calendering promotes, which is significant at low C-rate when intercalation occurs within the whole electrode volume, while it becomes irrelevant at high C-rate, when reaction takes place close to the separator due to $\mathrm{Li}^{+}$ion transport limitation in the electrolyte (as was observed in Fig. 3). Notably, the experimental curve is consistent with the predicted performance of the thick electrode in Fig. 8b; (3) for the intermediate mass loading, the capacity is identical between calendered and uncalendered samples until reaching $2 \mathrm{C}$, after which the rate capability loss due to compression becomes significant.

In summary, as manufacturing guidelines, calendering would not benefit the volumetric energy density of power-oriented low mass loading thin electrodes $\left(1 \mathrm{mAh} \mathrm{cm}^{-2}\right)$ due to the significant microstructural and reaction heterogeneity that is mainly caused by the large particle size; $20 \%$ calendering is feasible to improve the volumetric energy density with moderate rate capability for hybrid applications; higher calendering is recommended for energy-oriented cells to enhance the volumetric energy density further, at the cost of catastrophic capacity drop beyond $1 \mathrm{C}$. This unique insight into the manufacturing process is only possible with the new advances in imaging, and image-based modelling presented for the first time here.

\section{Discussion}

In this study, we addressed critical technical challenges both in advanced electrode design and engineering for different applications, assisted by the combination of X-ray CT-based techniques and experiments. The DSS technique that combines separate scans of high-attenuating NMC and low-attenuating CBD enables 

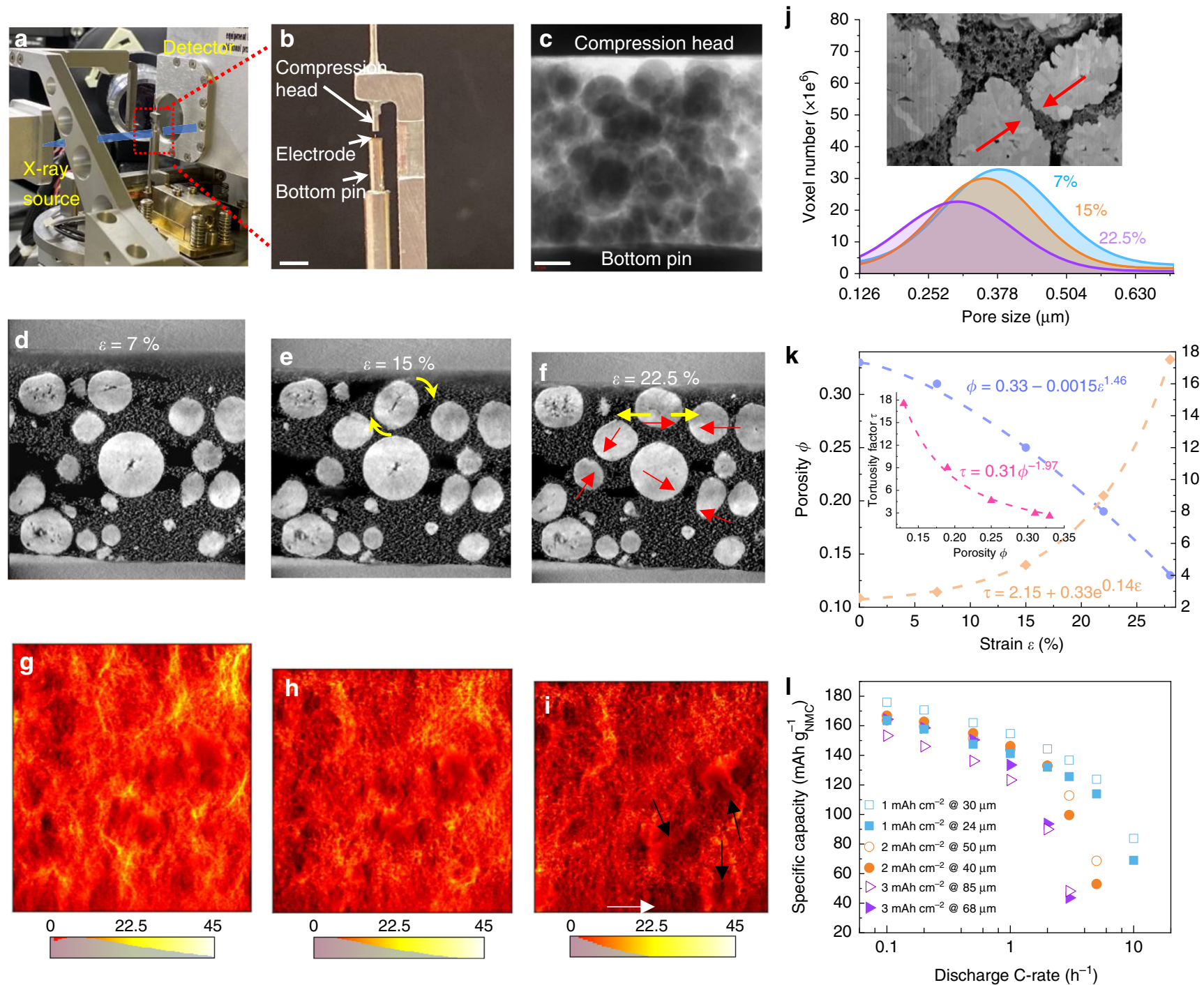

Fig. 9 Calendering effect on electrode microstructure and performance. a Experimental setup of the time-lapse X-ray nano-CT; $\mathbf{b}$ magnified image showing the details of the alignment; $\mathbf{c}$ X-ray radiograph before the compression; $\mathbf{d}-\mathbf{f}$ slices from nano-CT images showing the electrode microstructural evolution under incremental calendering steps; $\mathbf{g}-\mathbf{i}$ simulated mass flux map in the calendered pore structures respectively, with the colour legend overlaid by the flux histogram; $\mathbf{j}$ pore size distribution at incremental calendering steps. The inset displays the 'crushed' CBD and loss of porosity; $\mathbf{k}$ quantification of the microstructural parameters as a function of calendering steps based on the reconstructed 3D volumes; I experimental electrochemical performance comparison between electrodes of three mass loadings, calendered and uncalendered. The scale bar in (b, c) represents $2 \mathrm{~mm}$ and $10 \mu \mathrm{m}$ respectively.

us to reconstruct the $3 \mathrm{D}$ electrode including microstructural heterogeneities at nanoscale for the first time. The 3D microstructural-resolved electrochemical model highlighted that, macroscopically, the gradients of electrolyte concentration and SoL increase with C-rate and depth-of-discharge in the electrode through-thickness direction due to $\mathrm{Li}^{+}$ion transport limitation in the electrolyte. The heterogeneous particle and pore phase distribution lead to non-uniform reactivity and reactant transport, inducing uneven intercalation behaviour between particles, which causes underutilisation of capacity and reduced power density, particularly under high rate conditions. Graded-microstructure electrodes are shown to significantly improve the rate capability while maximising the accessible energy density at high mass loading as the porosity in the vicinity of the separator predominantly determines the high rate performance. Thicker electrodes show more reduced specific capacity due to mass transport limitations. Calendering has opposite effects on thin and thick electrodes: while it would not benefit the volumetric energy density of power-oriented thin electrodes due to the significant heterogeneity, 20\% calendering can improve the energy density with moderate rate capability for hybrid applications and higher calendering is recommended for energy-oriented cells, although at the cost of catastrophic capacity drop beyond $1 \mathrm{C}$. The DSS method and modelling techniques presented in this study are applicable across multiple length scales to many other advanced energy conversion and storage devices, such as supercapacitors and fuel cells, to provide new insights into the strategy of electrode design and optimisation.

\section{Methods}

Sample preparation and coin cell assembly. A mixture of $90 \mathrm{wt} \% \mathrm{LiNi}_{1 / 3} \mathrm{Mn}_{1 /}$ ${ }_{3} \mathrm{Co}_{1 / 3} \mathrm{O}_{2}$ (NMC111) (Targray, Canada), $5 \mathrm{wt} \%$ conductive carbon black (Timical Super C65, Imerys, Switzerland) and $5 \mathrm{wt} \%$ polyvinylidene fluoride (PVDF) (Arkema, France) was homogenised in a dual asymmetric centrifuge system (SpeedMixer DAC 150.1 FVZ-K, Hauschild, Germany). N-methyl-2-pyrrolidinone (NMP; anhydrous, Sigma-Aldrich) was used as a solvent. The slurry was cast onto a $20-\mu \mathrm{m}$-thick aluminium sheet and spread with a doctor blade. The electrode sheet was then initially dried in an oven prior to further drying in a vacuum oven overnight at $80^{\circ} \mathrm{C}$. The stand-alone CBD sample was prepared by mixing and 
homogenising $50 \mathrm{wt} \%$ conductive carbon black and $50 \mathrm{wt} \%$ PVDF with NMP as the solvent in an asymmetric centrifuge system, followed by casting and drying in the vacuum oven overnight at $80^{\circ} \mathrm{C}$. Electrode sheets with thicknesses of $50 \mu \mathrm{m}$ $\left(1.4 \mathrm{mAh} \mathrm{cm}^{-2}\right)$ and $100 \mu \mathrm{m}\left(2.8 \mathrm{mAh} \mathrm{cm}^{-2}\right)$ were prepared. The $50-\mu \mathrm{m}$-thick NMC cathode was punched to a diameter of $15 \mathrm{~mm}$ and assembled in coin cells (type CR2032) with a lithium anode and a Celgard 2325 separator under a protective argon atmosphere. One hundred microlitres of $1 \mathrm{M} \mathrm{LiPF}_{6}$ in EC/EMC (3:7 $\mathrm{v} / \mathrm{v}$ ) was used as the electrolyte.

Electrochemical testing. Discharge tests were performed on the assembled coin cells (three repeat tests) at room temperature using a potentiostat (Interface 1000E, Gamry Instruments or Maccor 4300) from 4.25 to $3 \mathrm{~V}$. The OCV of NMC111 vs. Li was measured using the Galvanostatic Intermittent Titration Technique (GITT, Supplementary Fig. 5). The coin cells were discharged at a constant current corresponding to a C-rate of $\mathrm{C} / 10$ in steps of $10 \%$ depth-of-discharge, followed by a pause at open circuit for $5 \mathrm{~h}$ to allow the voltage to relax prior to OCV measurement. This pulse/relaxation cycle was repeated until the $3 \mathrm{~V}$ cut-off voltage was reached.

X-ray computed tomography and 3D image analysis. An electrode disk of approximately $1 \mathrm{~mm}$ in diameter was punched from the same sheet of cathode material as described above, and then was mounted on a pin head using fast-setting epoxy. The electrode disk was reduced to a pillar of ca. $90 \mu \mathrm{m}$ in diameter using a micro-milling laser technique (A Series/Compact Laser Micromachining System, Oxford Lasers, Oxford, UK). The electrode pillar was then scanned using a labbased X-ray nano-CT system (Zeiss Xradia Ultra 810 X-ray microscope, Carl Zeiss, CA, USA $)^{43}$ at an isotropic voxel size of $126 \mathrm{~nm}$ and a field of view of $64 \mu \mathrm{m} \times 64$ $\mu \mathrm{m}$. A quasi-monochromatic X-ray beam with a $\mathrm{Cr}$ characteristic emission energy of $5.4 \mathrm{keV}$ was used and 2001 sequential projections with an exposure time of $14 \mathrm{~s}$ were collected over $180^{\circ}$ rotation. For the CBD imaging, a voxel size of $63 \mathrm{~nm}$ and exposure time of $40 \mathrm{~s}$ were used and the other parameters remained the same. The radiographic projections were automatically reference corrected, followed by the alignment of centre shifts (post collection). The corrected, aligned radiographs were reconstructed using standard, parallel beam, filtered-back projection algorithms $\mathrm{s}^{44}$, implemented in the Zeiss Scout and Scan software package (Carl Zeiss, CA, USA). The reconstructed 3D volume of the electrode was imported into commercial software package Avizo V9.4 (Avizo, Thermo Fisher Scientific, Waltham, Massachusetts, USA) for microstructural characterisation and tortuosity factor measurement, which can also be achieved by using open-source software TauFactor ${ }^{45}$. A detailed thresholding segmentation workflow and the validation are illustrated shown in Supplementary Fig. 1. The pore size distribution was analysed using the Local Thickness plug-in from the open-source software Fiji ${ }^{4}$. The shape and orientation of NMC particles were extracted by calculating the moments of inertia of its ellipsoid template and the eigenvalues of the covariance matrix ${ }^{47}$. The sphericity of the NMC particles is presented in terms of the ratio between the minor and major axes of their best-fit ellipsoid. The graded-microstructure electrodes were artificially obtained by morphological operation.

Morphological operation for graded-structures. The graded-structures were obtained by voxel manipulation based on the as-prepared 3D microstructure. Specifically, dilation and erosion were operated to enlarge and shrink the target phase by pushing or pulling the voxels at the phase boundary using a structure element (SE). The algorithms of these morphological operations can be found here $^{37,38}$. To obtain a graded-particle volume, the original particles were firstly eroded by approx. $2 \mu \mathrm{m}$. Small particles $(2-3 \mu \mathrm{m})$, which were directly taken from the as-prepared data, were spatially transformed (rotation, flip, etc.) and added into the non-particle region so as to maintain the same volume fraction of the active material, which can be quantified by image analysis tools in Avizo. For gradedporosity, all the boundary voxels of the pore phase in the respective modified region were pushed outward by $250 \mathrm{~nm}$ using dilation operation, while the voxels constituting the active materials were "locked" so that they cannot be affected, which means the mass loading of the electrode remains unchanged, and it is just the relative movement of the pore/CBD boundaries.

3D physics-based microstructure-resolved model. The reconstructed 3D volume of the NMC111 electrode $(43 \mu \mathrm{m} \times 43 \mu \mathrm{m} \times 50 \mu \mathrm{m})$ was first segmented into three phases (i.e. active material, pore and CBD) based on greyscale thresh olding (details about the thresholding workflow is shown in Fig. 3 and Supplementary Note 1), and was then imported into the commercial software package Simpleware ScanIP N-2018.03 for meshing. The physical size of the mesh element was adjusted according to the feature size of each phase and conformal nodes were ensured at the boundaries. The meshed volume consisted of 30 million tetrahedral elements and is shown in Supplementary Fig. 3.

The electrochemical model of the lithium metal half-cell was mathematically developed via a series of partial differential equations (PDEs) based on the generalised Poisson-Nernst-Planck (gPNP) equations ${ }^{48}$, which is a derivative of the Newman model ${ }^{49}$, implemented using Mathematics/General Form PDE module in the commercial software package COMSOL Multiphysics V5.3. Balance equations for the species $\mathrm{Li}^{+}, \mathrm{PF}_{6}{ }^{-}, \mathrm{e}^{-}$and $\mathrm{Li}$ were solved in the entire simulation domain. Concentrated solution theory and electro-neutrality were implemented to describe mass transport in the electrolyte ${ }^{48,50}$, while Fick's law was used for $\mathrm{Li}$ diffusion in the active material particles and Ohm's law for electron transport in the CBD phase and active material particles. The charge-transfer reaction follows a kinetic expression originating from non-equilibrium thermodynamics, resulting in a Butler-Volmer-type expression. Details of the physical equations, boundary conditions and input parameters are shown in Supplementary Fig. 4 and Supplementary Tables $1-3$. It is noted that no volume-averaged parameters (e.g. porosity and effective transport parameters) are used in the microstructureresolved model.

\section{Data availability}

All data presented in this manuscript are available from the corresponding author upon request.

\section{Code availability}

The code for the $3 \mathrm{D}$ electrochemical modelling is available from the corresponding author upon request.

Received: 24 December 2019; Accepted: 23 March 2020; Published online: 29 April 2020

\section{References}

1. Cano, Z. P. et al. Batteries and fuel cells for emerging electric vehicle markets. Nat. Energy 3, 279-289 (2018).

2. Kwade, A. et al. Current status and challenges for automotive battery production technologies. Nat. Energy 3, 290-300 (2018).

3. Albertus, P., Babinec, S., Litzelman, S. \& Newman, A. Status and challenges in enabling the lithium metal electrode for high-energy and low-cost rechargeable batteries. Nat. Energy 3, 16-21 (2018).

4. Qian, J. et al. High rate and stable cycling of lithium metal anode. Nat. Commun. 6, 6362 (2015).

5. Shearing, P. R., Howard, L. E., Jørgensen, P. S., Brandon, N. P. \& Harris, S. J. Characterization of the 3-dimensional microstructure of a graphite negative electrode from a Li-ion battery. Electrochem. Commun. 12, 374-377 (2010).

6. Otagawa, T., Jina, A. N. \& Madou, M. J. Google Patents. 5,294,504 (1994).

7. Stephenson, D. E. et al. Modeling 3D microstructure and ion transport in porous Li-ion battery electrodes. J. Electrochem. Soc. 158, A781-A789 (2011).

8. Dillon, S. J. \& Sun, K. Microstructural design considerations for Li-ion battery systems. Curr. Opin. Solid State Mater. Sci. 16, 153-162 (2012).

9. Sun, Y.-K. et al. Nanostructured high-energy cathode materials for advanced lithium batteries. Nat. Mater. 11, 942 (2012).

10. Liu, J. et al. A flexible quasi-solid-state nickel-zinc battery with high energy and power densities based on 3D electrode design. Adv. Mater. 28, 8732-8739 (2016).

11. $\mathrm{Xu}, \mathrm{Y}$. et al. In situ visualization of state-of-charge heterogeneity within a $\mathrm{LiCoO} 2$ particle that evolves upon cycling at different rates. ACS Energy Lett. 2, 1240-1245 (2017).

12. Harris, S. J. \& Lu, P. Effects of inhomogeneities-nanoscale to mesoscale-on the durability of Li-ion batteries. J. Phys. Chem. C 117, 6481-6492 (2013).

13. Finegan, D. P. et al. In-operando high-speed tomography of lithium-ion batteries during thermal runaway. Nat. Commun. 6, 6924 (2015).

14. Nanda, J. et al. Local state-of-charge mapping of lithium-ion battery electrodes. Adv. Funct. Mater. 21, 3282-3290 (2011).

15. Forouzan, M. M., Mazzeo, B. A. \& Wheeler, D. R. Modeling the effects of electrode microstructural heterogeneities on Li-ion battery performance and lifetime. J. Electrochem. Soc. 165, A2127-A2144 (2018).

16. Yu, X. et al. Simultaneous operando measurements of the local temperature, state of charge, and strain inside a commercial lithium-ion battery pouch cell. J. Electrochem. Soc. 165, A1578-A1585 (2018).

17. Taiwo, O. O. et al. Comparison of three-dimensional analysis and stereological techniques for quantifying lithium-ion battery electrode microstructures. $J$. Microsc. 263, 280-292 (2016).

18. Zhu, Y. et al. Fast lithium growth and short circuit induced by localizedtemperature hotspots in lithium batteries. Nat. Commun. 10, 2067 (2019).

19. Chen, G., Song, X. \& Richardson, T. J. Electron microscopy study of the $\mathrm{LiFePO} 4$ to FePO4 phase transition. Electrochem. Solid-State Lett. 9, A295-A298 (2006).

20. Tang, M., Carter, W. C. \& Chiang, Y.-M. Electrochemically driven phase transitions in insertion electrodes for lithium-ion batteries: examples in lithium metal phosphate olivines. Annu. Rev. Mater. Res. 40, 501-529 (2010).

21. Smith, A., Burns, J. \& Dahn, J. A high precision study of the Coulombic efficiency of Li-ion batteries. Electrochem. Solid-State Lett. 13, A177-A179 (2010). 
22. Shim, J. \& Striebel, K. A. Effect of electrode density on cycle performance and irreversible capacity loss for natural graphite anode in lithium-ion batteries. $J$. Power Sources 119, 934-937 (2003).

23. Müller, S. et al. Quantifying inhomogeneity of lithium ion battery electrodes and its influence on electrochemical performance. J. Electrochem. Soc. 165, A339-A344 (2018).

24. Liu, Y., Zhu, Y. \& Cui, Y. Challenges and opportunities towards fast-charging battery materials. Nat. Energy 4, 540-550 (2019).

25. Finegan, D. P. et al. Characterising the structural properties of polymer separators for lithium-ion batteries in 3D using phase contrast X-ray microscopy. J. Power Sources 333, 184-192 (2016).

26. Garcia, R. E., Chiang, Y.-M., Carter, W. C., Limthongkul, P. \& Bishop, C. M. Microstructural modeling and design of rechargeable lithium-ion batteries. J. Electrochem. Soc. 152, A255-A263 (2005).

27. Yang, F. et al. Nanoscale morphological and chemical changes of high voltage lithium-manganese rich NMC composite cathodes with cycling. Nano Lett. 14, 4334-4341 (2014).

28. Hendricks, C., Williard, N., Mathew, S. \& Pecht, M. A failure modes, mechanisms, and effects analysis (FMMEA) of lithium-ion batteries. J. Power Sources 297, 113-120 (2015).

29. Birkl, C. R., Roberts, M. R., McTurk, E., Bruce, P. G. \& Howey, D. A. Degradation diagnostics for lithium ion cells. J. Power Sources 341, 373-386 (2017).

30. Rodrigues, M.-T. F. et al. A materials perspective on Li-ion batteries at extreme temperatures. Nat. Energy 2, 17108 (2017).

31. Ovejas, V. J. \& Cuadras, A. State of charge dependency of the overvoltage generated in commercial Li-ion cells. J. Power Sources 418, 176-185 (2019).

32. Newman, J. \& Tiedemann, W. Porous-electrode theory with battery applications. AICHE J. 21, 25-41 (1975).

33. Ebner, M., Chung, D. W., García, R. E. \& Wood, V. Tortuosity anisotropy in lithium-ion battery electrodes. Adv. Energy Mater. 4, 1301278 (2014).

34. Kehrwald, D., Shearing, P. R., Brandon, N. P., Sinha, P. K. \& Harris, S. J. Local tortuosity inhomogeneities in a lithium battery composite electrode. J. Electrochem. Soc. 158, A1393-A1399 (2011).

35. Dai, Y. \& Srinivasan, V. On graded electrode porosity as a design tool for improving the energy density of batteries. J. Electrochem. Soc. 163, A406-A416 (2016).

36. Ramadesigan, V., Methekar, R. N., Latinwo, F., Braatz, R. D. \& Subramanian, V. R. Optimal porosity distribution for minimized Ohmic drop across a porous electrode. J. Electrochem. Soc. 157, A1328-A1334 (2010).

37. Höhne, K. H. \& Hanson, W. A. Interactive 3D segmentation of MRI and CT volumes using morphological operations. J. Comput. Assist. Tomogr. 16, 285-294 (1992).

38. Chudasama, D., Patel, T., Joshi, S. \& Prajapati, G. Image segmentation using morphological operations. Int. J. Comput. Appl. 117, 16-19 (2015).

39. Huang, C. \& Grant, P. S. Coral-like directional porosity lithium ion battery cathodes by ice templating. J. Mater. Chem. A 6, 14689-14699 (2018).

40. Gaudillere, C. \& Serra, J. M. Freeze-casting: fabrication of highly porous and hierarchical ceramic supports for energy applications. Bol. Soc. Esp. Ceram. V 55, 45-54 (2016)

41. Tian, R. et al. Quantifying the factors limiting rate performance in battery electrodes. Nat. Commun. 10, 1933 (2019).

42. Patterson, B. M. et al. In situ laboratory-based transmission X-ray microscopy and tomography of material deformation at the nanoscale. Exp. Mech. 56, 1585-1597 (2016).

43. Schurch, R., Rowland, S. M., Bradley, R. S. \& Withers, P. J. Comparison and combination of imaging techniques for three dimensional analysis of electrical trees. IEEE Trans. Dielectr. Electr. Insul. 22, 709-719 (2015).

44. Singh, S. et al. Abdominal CT: comparison of adaptive statistical iterative and filtered back projection reconstruction techniques. Radiology 257, 373-383 (2010).

45. Cooper, S. J., Bertei, A., Shearing, P. R., Kilner, J. A. \& Brandon, N. P. TauFactor: an open-source application for calculating tortuosity factors from tomographic data. SoftwareX 5, 203-210 (2016).

46. Schindelin, J. et al. Fiji: an open-source platform for biological-image analysis. Nat. Methods 9, 676-682 (2012).

47. Campbell, R. J. \& Flynn, P. J. Eigenshapes for 3D object recognition in range data. Proc. IEEE Comput. Soc. Conf. Comput. Vis. Pattern Recogn. 2, 505-510 (1999).

48. Lai, W. \& Ciucci, F. Mathematical modeling of porous battery electrodesrevisit of Newman's model. Electrochim. Acta 56, 4369-4377 (2011).
49. Doyle, M., Fuller, T. F. \& Newman, J. Modeling of galvanostatic charge and discharge of the lithium/polymer/insertion cell. J. Electrochem. Soc. 140 , 1526-1533 (1993)

50. Cai, L. \& White, R. E. Mathematical modeling of a lithium ion battery with thermal effects in COMSOL Inc. Multiphysics (MP) software. J. Power Sources 196, 5985-5989 (2011).

\section{Acknowledgements}

This work was supported by the Engineering and Physical Sciences Research Council (EP/R020973/1, EP/M028100/1) and the Faraday Institution (faraday.ac.uk; EP/S003053/ 1, grant numbers FIRG003 and FIRG001). P.R.S. acknowledges funding from the Royal Academy of Engineering (CiET1718\59). X.L. and G.H. acknowledge support from the National Measurement System of the UK Department of Business, Energy and Industrial Strategy. Dr. Vladimir Yufit and Dr. Farid Tariq (Addionics Ltd.) are gratefully acknowledged for preliminary discussions. This work was authored in part by the National Renewable Energy Laboratory, operated by Alliance for Sustainable Energy, LLC, for the U.S. Department of Energy (DOE) under Contract No. DE-AC3608GO28308. Funding was provided by the U.S. Department of Energy Office of Energy Efficiency and Renewable Energy Vehicle Technologies Office. The views expressed in the article do not necessarily represent the views of the DOE or the U.S. Government. The U.S. Government retains and the publisher, by accepting the article for publication, acknowledges that the U.S. Government retains a nonexclusive, paid-up, irrevocable, worldwide license to publish or reproduce the published form of this work, or allow others to do so, for U.S. Government purposes.

\section{Author contributions}

X.L., A.B. and P.R.S. conceived the study. X.L. and A.B. conducted the X-ray CT scans, image analysis, modelling and drafted the manuscript. D.P.F. provided additional X-ray CT data and analysis. S.R.D. contributed to the in situ X-ray CT scans. C.T., J.S.W. and T. M.M.H. supported the electrochemical test of the cells. K.B.O. and E.K. performed the rate capability test of the calendered cells; X.L., A.B., D.P.F., C.T., S.R.D., J.S.W., T.M.M.H., K.B.O., G.H., E.K., D.J.L.B. and P.R.S. reviewed the manuscript.

\section{Competing interests}

The authors declare no competing interests.

\section{Additional information}

Supplementary information is available for this paper at https://doi.org/10.1038/s41467020-15811-x.

Correspondence and requests for materials should be addressed to P.R.S.

Peer review information Nature Communications thanks Kang Dong, researcher Robert Kee, Jagjit Nanda and the other, anonymous, reviewer(s) for their contribution to the peer review of this work. Peer reviewer reports are available.

Reprints and permission information is available at http://www.nature.com/reprints

Publisher's note Springer Nature remains neutral with regard to jurisdictional claims in published maps and institutional affiliations.

Open Access This article is licensed under a Creative Commons Attribution 4.0 International License, which permits use, sharing, adaptation, distribution and reproduction in any medium or format, as long as you give appropriate credit to the original author(s) and the source, provide a link to the Creative Commons license, and indicate if changes were made. The images or other third party material in this article are included in the article's Creative Commons license, unless indicated otherwise in a credit line to the material. If material is not included in the article's Creative Commons license and your intended use is not permitted by statutory regulation or exceeds the permitted use, you will need to obtain permission directly from the copyright holder. To view a copy of this license, visit http://creativecommons.org/ licenses/by/4.0/

(c) The Author(s) 2020 